\title{
PARAPARESIA POR GOTA
}

\author{
ADELMO FERREIRA * - BONFIM ALVES SILVA Jr*** - F. MENEZES BRAGA :** \\ NOBIA M. NOSCHESE GARGIULO **** _ J. NORBERTO STAVALE*****
}

RESUMO - Os autores apresentam um caso de bloqueio de canal raquidiano por tofo em paciente de 23 anos de idade com gota tofácea crônica e artrite gotosa, que desenvolveu paraparesia crural. Submetido a laminectomia, com exérese de acúmulos de cristais junto ao lígamento amarelo, não houve melhora do déficit motor. A revisão da literatura mostrou 9 casos previamente relatados.

\section{Paraparesis in gout.}

SUMMARY - A case of a 23 year-old-man with a history of polyarthritis and chronic tophaceous gout is reported. He developed a crural paraparesis caused by sodium urate deposits in the spinal canal. Decompressive laminectomy and removal of urate-laden ligamentum flavum was performed without improvement. According to the literature there are nine cases previously reported.

A gota é uma das doenças de relato mais antigo nos escritos médicos. Já era conhecida por Hipocrates no século V a.C.; no século III d.C., Galeno fez referências ao tofo. Em 1797, Wollaston descreveu a presença de urato no tofo 2,3 .

$O$ achado cirúrgico de tofo em localização rara, no canal raquidiano, motivou-nos à revisão da literatura e a relatar éste caso.

\section{OBSERVAÇAOO}

GNV, paciente com 23 anos, masculino, pardo, admitido em 13-11-87 com história de gota tofácea crônica e de artrite gotosa desde os 17 anos de idade, em uso irregular de allopurinol. Vinte dias antes da admissão passou a queixar-se de perda progressiva da força nos membros inferiores (MMII) e dificuldade à miç̧ão. Ao exame apresentava tofos (um deles infectado) nas articulaçôes metatarso-falangeanas, interfalangeanas e cotovelo; paraparesia crural espástica (força muscular grau 2 nos MMII; nível de sensibilidade em T4. Exames complementares - Dosagem de ácido úrico no sangue: $14 \mathrm{mg} / \mathrm{dl}$ RX de mãos e pés: presença de erosão em 'saca bocado' e imagens sugestivas de tofos nas articulações. RX de coluna: sinais de ósteo-artrite difusa. Liqüido cefalorraquidiano lombar: proteínas $206 \mathrm{mg} / \mathrm{dl}$; suspeita de bloqueio de canal às provas de permeabilidade. Perimielografia: bloqueio parcial de C7 a T5 (Fig. 1). Foi submetido a laminectomia (C6-T5), encontrando-se ao nivel de T4-T5 abscesso de cápsula fina que ocupava a parte posterior do canal no espaço

Hospital Municipal Arthur Ribeiro de Saboya (HMARS) e Escola Paulista de Medicina (EPM): * Pós-Graduando enı Neurocirurgia EPM, Médico do Serviço de Neurocirurgia (SN) HMARS; ** Pós-Graduando em Fisiologia Humana no Instituto de Ciências Biomédicas da Universidade de São Paulo, Médico do SNHMARS; ** Professor Adjunto de Neurocirurgia, Chefe da Disciplina de Neurocirurgia e Coordenador do Curso de Pós-Graduação em Neurocirurgia, EPM; **** Médica do SNHMARS; ****: Professor Adjunto da Disciplina de Anatomia Patológica, EPM. 
extradural e contínha líquitido espesso de cor amarelo-clara em seu interior. Feita drenagem, foi lavado o local com solução de ampicilina. De $\mathrm{C} 7$ e $\mathrm{T} 4$ foram retirados acúmulos de cristais de cor clara, de tamanho variável, distribuídos irregularmente ao redor do saco dural, comprimindo-o principalmente na sua face posterior, fundindo-se com o ligamento amarelo (Fig. 2). Observou-se retorno da pulsação do saco dural após essa descompressão. Foram removidos parte do pedículo e da faceta de $T 3$, que se encontravam espessados e esbranquiçados, envolvidos pela lesão. Não foi aberta a dura-máter, pois o acometimento aparentava ser somente extradural e haveria contaminação pelo abscesso encontrado, cuja bacterioscopia revelou presença de cocos Gram positıvos. O exame anátomo-patológico do material contendo cristais mostrou-se compatível a gota (Fig. 3). Recebeu no pós-operatório colchicina e, posteriormente, foi mantido com allopurinol. Durante a internação desenvolveu escaras de decúbito, infeção urinária e hemorragia digestiva por úlcera gástrica, tratada cirurgicamente. Recebeu alta sem modificação de seu quadro neurológico.

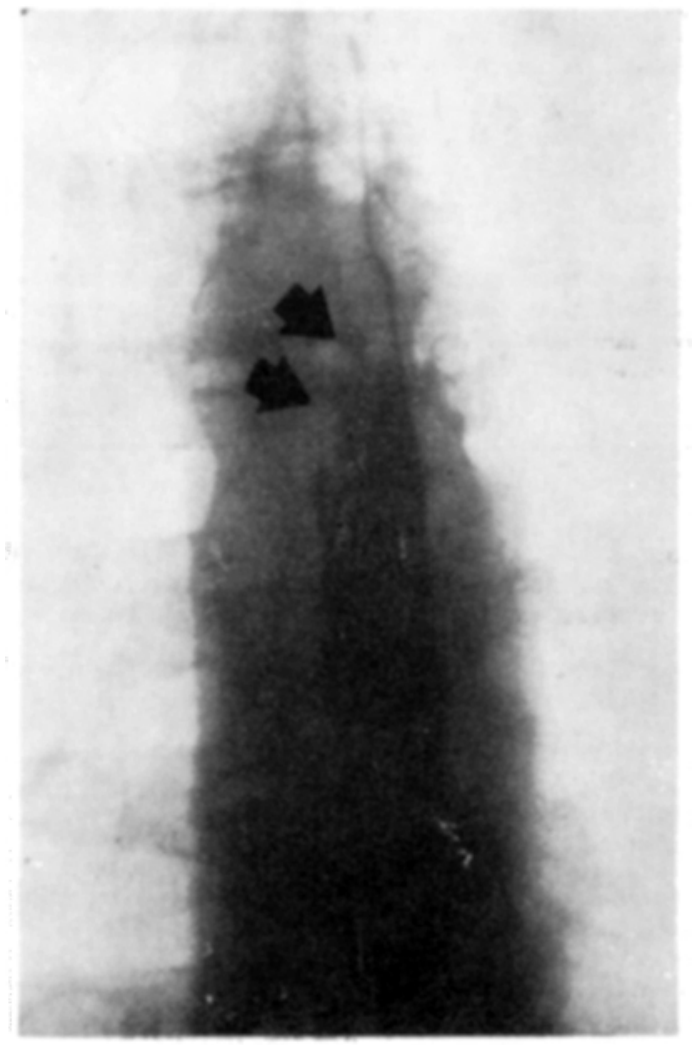

Fig. 1 - Caso GNV. Perimielografia ascendente mostrando o limite inferior do bloqueio parcial em T5. Nota-se a passagem de pequena quantidade de contraste pelo lado esquerdo do canal (setas).

\section{COMENTARIOS}

A gota pode apresentar-se sob as formas de hiperuricemia assintomática, artrite gotosa aguda ou de gotatofácea crônica, podendo mais de uma forma ocorrer em um mesmo paciente. $O$ aspecto característico da gota crônica é a deposição de urato monossódico nas articulaçōes e no tecido conjuntivo peri-articular. Tofos são tipicamente encontrados no esqueleto apendicular, sendo a ocorrência no esqueleto axial incomum 14. Tem sido relatado o depósito de urato em vários locais do organismo 6,9 , inclusive no cérebro, em que Sass 11 descreveu, em um caso, acúmulo de grânulos com coloração positiva para urato. A compressão de nervo periférico por tofo também é descrita 8 .

$\mathrm{Na}$ tabela 1 são mostrados os casos de gota no canal raquidiano descritos na literatura 4,5,7,8,10,12-15. Além destes, Aaron 1 descreveu um caso de provável gota na coluna cervical; entretanto, o diagnóstico não chegou a ser confirmado patologica- 


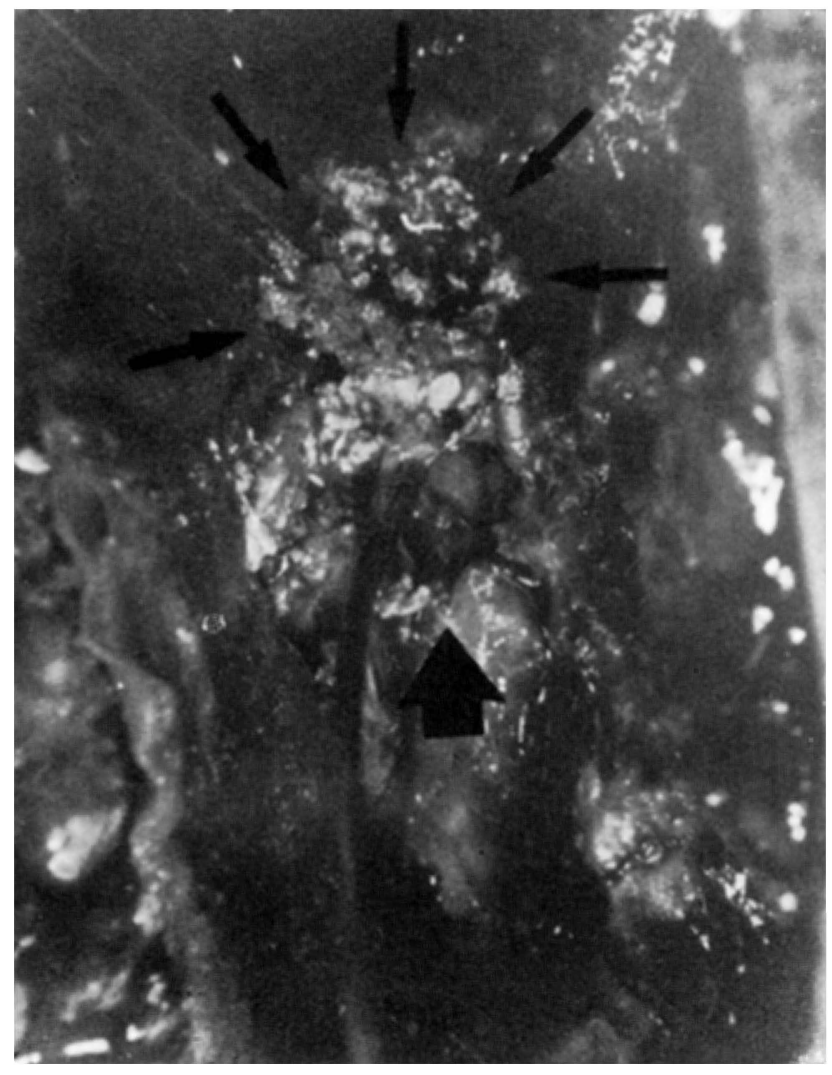

Fig. 2 - Caso GNV. Laminectomia torácica mostrando o tofo (setas acima) sendo descolado e levantado do saco dural (seta abaixo).

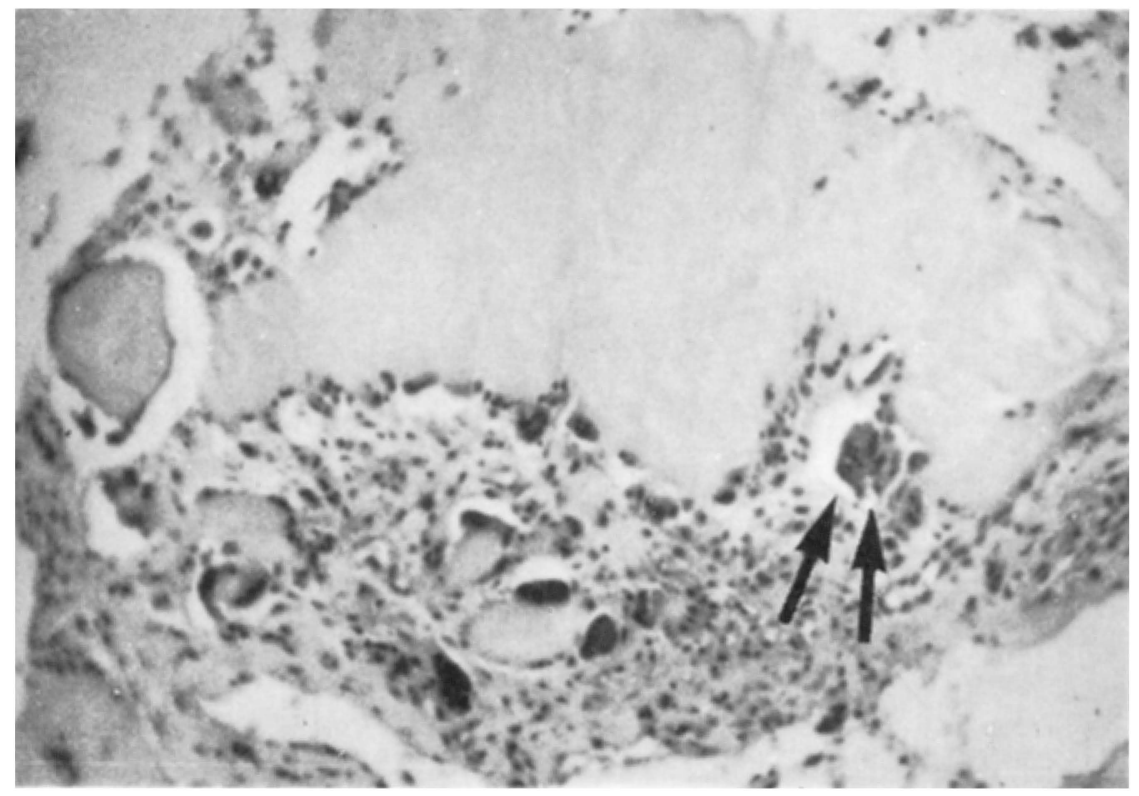

Fig. : - Caso GNV. Presença de celulas gigantes multinucleadas de tipo corpo estranho (seta), ao redor de material amorfo, acelular e avascular (HE. 160×). 


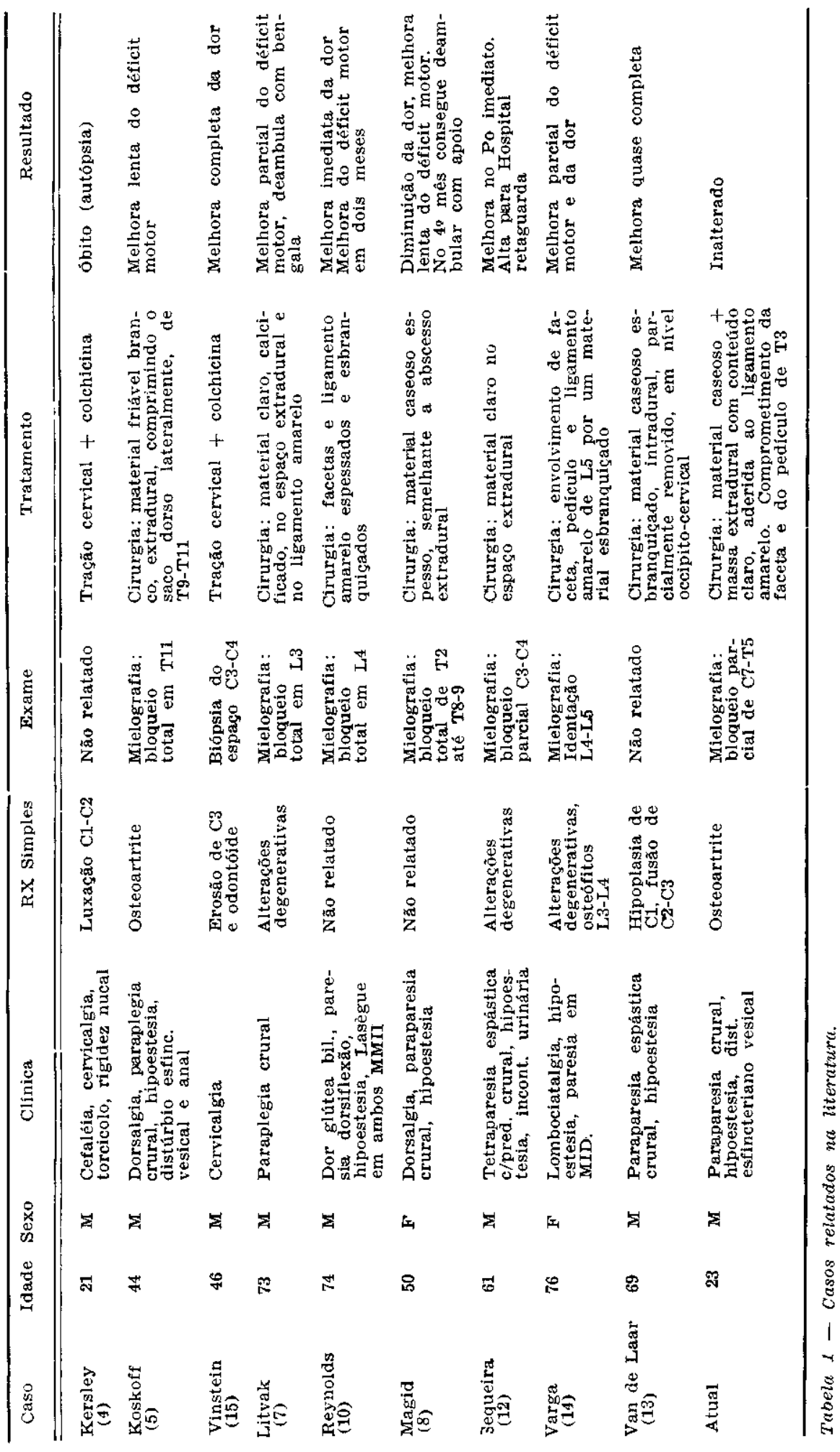


mente. A média de idade de acometimento foi 53 anos, tendo predominado o sexo masculino. A coluna cervical foi a mais acometida. Clinicamente, o tofo pode simular hérnia discal ou tumor ${ }^{14}$, sendo o déficit motor de ocorrência frequente. A compressão pode ocorrer pelo tofo, pelo abscesso (ambos presentes neste caso) ou por instabilidade óssea consequente a erosão 4,15. No caso aqui descrito havia envolvimento de parte do pediculo e da faceta, mas sem causar erosão. O ligamento amarelo comumente está invadido, com aspecto de espessamento. Lesão intradural foi encontrada por van de Laar 13 , mas a maioria dos autores năo faz referência a investigação intradural no ato operatório, talvez por estarem convencidos de que o achado extradural já seria suficiente para explicar o quadro apresentado por seus pacientes. Além disso, quando há presença de material infectado, como ocorreu neste caso, existe 0 risco de se levar a contaminação para o espaço intradural. Dois casos foram submetidos a tratamento clínico e, em ambos, o quadro predominante era dor local; aqueles casos nos quais exames subsidiários mostraram compressão evidente foram abordados cirurgicamente e, após, submetidos a tratamento medicamentoso (colchicina/allopurinol).

A revisão da literatura apresentada nos alerta para a necessidade da lembrança do diagnóstico de tofo em pacientes com gota e que apresentem sintomas neurológicos.

\section{REFERENCIAS}

1. Aaron SL, Miller JDR, Percy JS - Tophaceous gout in the cervical spine. J Rheumatoi $11: 862,1984$.

2. Hall MC, SeIin G - Spinal involvement in gout: a case report with autopsy. J Bone Joint Surg 42A:341, 1960.

3. Kelley WN, Fox IH - Gout and related disorders of purine metabolism. In Kelley WN, Harris JR. Ruddy S, Sledge CB (eds): Textbook of Rheumatology. Ed 2. Saunders, Philadelphia, pg 1359.

4. Kersley GD, Mandel L, Jeffrey MR - Gout: an unusual case with softening and subluxation of the eirat cervical vertebra and splenomegalla. Ann Rheum Dis 9:282, 1950 .

5. Koskoff YD, Morris LE, Lubic LG - Paraplegia as a complication of gout. J Am Med Assoc 152:37, 1853 .

6. Lichtenstein L, Scott HW, Levin MH - Pathologic changes in gout: survey of eleven necropsied cases. Am J Pathol 32:871, 1956.

7. Litvak J, Briney W - Extradural spinal depositions of urates producing paraplegia: case report. J Neurosury, $39: 656,1973$.

8. Magid SK, Gray GE, Anand A - Spinal cord compression by tophi in a patient with chronic polyarthritis: case report and literature review. Arthr Rheum 24:1431, 1981.

9. Malawista SE, Seegmiller JE, Hathway BE, Sokoloff L $\longrightarrow$ Sacroiliac gout. J Am Med Assoc 194:106, 1965 .

10. Reynolds AF Jr, Wyler AR, Norris HT - Paraparesis secondary to sodium urate deposits in the ligamentum flavum. Arch Neurol $33: 795,1976$.

11. Sass JK, Itabashi HH, Dexter RA - Juvenile gout with brain involvement. Arch Neurol $13: 639,1965$.

12. Sequeira W, Bouffard A, Salgia K, Skosey $J$ - Quadriparesis in tophaceous gout. Arthr Rheum 24:1428, 1981.

13. van de Laar MAFJ, van Soesbergen RM, Matricali B - Tophaceous gout of the cervical spine without peripheral tophi. Arthr Rheum 30:237, 1987.

14. Varga J, Giampaolo C, Goldenberg DL - Tophaceous gout of the spine in a patient with no peripheral toph: case report and revlew of the literature. Arthr Rheum 28:1312, 1985 .

15. Vinstein AL, Cockerill EM - Involvement of the spine in gout: a case report. Radiology 103:311, 1972. 